\title{
Avaliação da qualidade do cuidado hospitalar no Brasil: uma revisão sistemática
}

\author{
Quality assessment of hospital care in Brazil: \\ a systematic review
}

\author{
Evaluación de la calidad de la atención hospitalaria \\ en Brasil: una revisión sistemática
}

\author{
${ }_{1}^{1}$ Agência Nacional de Saúde \\ Suplementar, Rio de Janeiro, \\ Brasil. \\ 2 Escola Nacional de Saúde \\ Pública Sergio Arouca, \\ Fundação Oswaldo Cruz, \\ Rio de Janeiro, Brasil. \\ Correspondência \\ J. P. Machado \\ Agência Nacional de Saúde \\ Suplementar. \\ Av. Augusto Severo 84, 10 o \\ andar, Rio de Janeiro, $R J$ \\ 20021-040, Brasil. \\ juliana.pm@gmail.com
}

\begin{abstract}
Quality assessment of hospital services has drawn growing international attention, driven by demand from funders, providers, practitioners, and patients. The objective of this study was to review the literature on hospital quality assessment in Brazil and analyze the main approaches, methodologies, and indicators used in the studies. The research design was a systematic literature review of scientific articles and doctoral and Master's theses published from 1990 to 2011. The review identified 2,169 documents, and 62 were included in the review, representing 48 separate studies. Most studies used secondary data and analyzed effectiveness, adequacy, safety, and efficiency, emphasizing the application of mortality rate, adequacy rate, adverse events rate, and length of stay. Methods to control differences in patient risks were mostly applied. This review identified central elements for both the development of this theme and the improvement of hospital care in Brazil.
\end{abstract}

Health Care Quality Evaluation; Hospital Services; Health Care Quality Indicators
Juliana Pires Machado ${ }^{1}$ Ana Cristina Marques Martins 1 Mônica Silva Martins 2

\section{Resumo}

A avaliação da qualidade de serviços hospitalares vem ganhando importância no mundo, sendo impulsionada pela demanda de financiadores, prestadores, profissionais e pacientes. $O$ objetivo deste estudo é revisar a literatura sobre estudos de avaliação da qualidade hospitalar no Brasil e analisar as principais abordagens, metodologias e indicadores utilizados. Foi aplicada revisão sistemática de artigos científicos, dissertações e teses com análises empíricas sobre o tema, publicados entre 1990 e 2011. Foram identificados 2.169 documentos e incluídos 62 documentos na revisão, que representam 48 estudos distintos. Predominou o uso de fontes de dados secundárias com análise das dimensões efetividade, adequação, segurança e eficiência, destacando-se a aplicação da taxa de mortalidade, taxa de adequação, taxa de eventos adversos e tempo de permanência. Métodos que controlam diferenças de risco dos pacientes foram majoritariamente aplicados. Busca-se com esta revisão contribuir apontando elementos centrais para o desenvolvimento do tema no país e para a qualificação do cuidado prestado.

Avaliação da Qualidade dos Cuidados de Saúde; Serviços Hospitalares; Indicadores de Qualidade em Assistência à Saúde 


\section{Introdução}

A avaliação da qualidade de serviços ganhou importância na área da saúde em todo o mundo, sendo impulsionada pela demanda de financiadores, prestadores, profissionais e do público. O desenvolvimento e a implementação de metodologias nessa área têm sido recorrentes e relacionam-se com os esforços para garantir transparência nos gastos, controlar os custos assistenciais crescentes, prestar cuidados adequados e equânimes e reduzir variações na prática clínica 1,2,3. Dada a natureza e especificidade dos processos organizacionais envolvidos, o ambiente hospitalar se destaca, pois concentra os maiores custos e a maior complexidade no cuidado prestado aos pacientes 4,5 .

Há cerca de 25 anos, o monitoramento de desempenho clínico no cuidado hospitalar ganhou espaço nas agendas de diferentes atores mundialmente, tendo, como marco, a publicação das taxas de mortalidade hospitalar, em 1986, nos Estados Unidos, e, em 1988, na Inglaterra 6,7, passando gradativamente a incorporar indicadores do processo de cuidado e, mais recentemente, indicadores sobre a segurança do paciente 8,9 .

Em outros países, inúmeras iniciativas de avaliações comparativas da qualidade de serviços hospitalares têm sido implementadas, com propostas metodológicas e uso de indicadores variados 1,2,10,11. Para além da finalidade científica e acadêmica, o resultado de pesquisas e a publicação de listas ranqueando os hospitais compõem programas de melhoria da qualidade e, em alguns casos, são utilizados como subsídio para a adoção de medidas regulatórias, muitas vezes, atrelando-se a sistemas de pagamento vinculados à qualidade 12,13,14 .

Enquanto o debate sobre a qualidade dos serviços hospitalares estende-se desde o início do século XX em países como os Estados Unidos e a Inglaterra, no Brasil, datam dos anos 1990 as primeiras iniciativas voltadas para a qualidade do cuidado 15,16. Destacam-se a criação da Comissão Nacional de Qualidade e Produtividade em Saúde, no âmbito do Programa Brasileiro de Qualidade e Produtividade, em 1994, para a formulação das diretrizes da estratégia de garantia de qualidade; a produção de consensos e diretrizes clínicas pelas sociedades profissionais a partir de 1996; a publicação do Manual de Normas para Hospitais em 1998 e a formação do Consórcio Brasileiro para Acreditação (CBA) em 1998 17,18.

No país, é recente a implantação de um sistema de acompanhamento da qualidade dos serviços de saúde que se proponha, formal e rotineiramente, a avaliar o desempenho dos estabelecimentos. Em 2005, o Programa Nacional de Avaliação de Serviços de Saúde (PNASS) foi lançado como instrumento de avaliação vinculado ao repasse de verbas aos gestores locais 19 . Em curso desde 2011, no âmbito do Governo Federal, há a proposta do programa de avaliação para a qualificação do Sistema Único de Saúde pelo Ministério da Saúde, com a publicação do índice de qualidade do SUS (ID-SUS) 20,21. No que tange às agências reguladoras que atuam na área de saúde, temos a divulgação da pontuação por qualidade das operadoras de planos privados de saúde, desde 2004, pela Agência Nacional de Saúde Suplementar (ANS) 22, que inclui, na avaliação das empresas, a dimensão assistência à saúde, além do programa de acreditação e as ações voltadas para a segurança do paciente $\mathrm{e}$ da qualidade em serviços de saúde desenvolvidas pela Agência Nacional de Vigilância Sanitária (ANVISA) 23,24.

No âmbito dos serviços, há ainda algumas iniciativas voltadas para a avaliação e melhoria da qualidade dos serviços de saúde, como a acreditação hospitalar, a certificação de estabelecimentos e as análises de satisfação do paciente 25. Por parte da academia, a matriz conceitual para avaliação de desempenho do sistema de saúde brasileiro, construída no âmbito do Projeto de Avaliação do Desempenho do Sistema de Saúde (PRO-ADESS; http://www.proadess.cict. fiocruz.br), apresenta um arcabouço teórico robusto para a investigação e o monitoramento do desempenho dos serviços e de fatores intervenientes 26,27 .

Neste trabalho, o objetivo compreendeu revisar sistematicamente a literatura em saúde pública sobre estudos empíricos de avaliação da qualidade do cuidado hospitalar realizados no Brasil, a fim de analisar as principais abordagens, metodologias e indicadores utilizados no país.

\section{Metodologia}

Na revisão sistemática, foram selecionados artigos científicos, dissertações e teses que examinaram a qualidade do cuidado prestado em hospitais brasileiros no período entre janeiro de 1990 e dezembro de 2011. Foram incluídos estudos empíricos cujo cenário era hospitalar e que, em suas análises, faziam referência à avaliação da qualidade de serviços ou ao desempenho hospitalar. Foram excluídos estudos teóricos sobre o campo da avaliação de serviços, estudos voltados aos métodos de avaliação da qualidade, análises de qualidade da atenção primária ou da gestão hospitalar, análises do desempenho do sistema de saúde, estudos sobre acesso, estudos relacionados à equidade, estudos que investigavam a 
conformidade na adesão a protocolos clínicos ou a adequação técnico-científica de procedimentos específicos, bem como estudos sobre avaliação de tecnologias, estudos descritivos da morbidade hospitalar ou de fatores de risco e estudos que não utilizavam dados de hospitais brasileiros. Não foram aplicados filtros para selecionar especificamente hospitais públicos ou privados, sendo incluídos todos aqueles que se adequavam às estratégias de busca, independentemente de sua natureza jurídica ou fonte de financiamento das internações que realiza (SUS, plano de saúde, particular).

Para o levantamento da literatura, foram consultadas as bases bibliográficas eletrônicas MEDLINE (interface PubMed), LILACS (Literatura Latino-Americana e do Caribe em Ciências da Saúde), SciELO (Scientific Electronic Library Online) e o banco de teses da Coordenação de Aperfeiçoamento de Pessoal de Nível Superior (Capes). Algumas bases de dados de universidades brasileiras foram consultadas; no entanto, não foram utilizados estudos obtidos nessas buscas, uma vez que, em sua maioria, tais bases remetem o pesquisador à própria busca da Capes ou da Biblioteca Virtual em Saúde (BVS), as quais já haviam sido consultadas neste estudo.

Para composição da estratégia de busca, com base nos descritores genéricos dos termos da área da avaliação e nos descritores referentes às dimensões da qualidade de interesse, conforme o PRO-ADESS (dando destaque às dimensões efetividade, eficiência, respeito ao direito, aceitabilidade, continuidade, segurança), selecionaram-se termos do MeSH (Medical Subject Heading Terms), que foram aplicados na busca do MEDLINE. Esses mesmos termos serviram de ponto de partida para a definição das estratégias de busca usadas nas demais bases. Para a base de teses Capes, a estratégia precisou ser seccionada e aplicada em partes, devido à indisponibilidade de mecanismo avançado de busca. As palavraschave foram pesquisadas em português e inglês, não tendo sido utilizados limites para a língua de apresentação dos estudos.

Para tratar as duplicidades de artigos recuperados nas diferentes bases, os documentos originalmente encontrados em cada uma delas foram ordenados pelo título e pelo primeiro autor, sendo excluídos aqueles que apareciam mais de uma vez. Manteve-se, como informação da fonte de pesquisa, aquela em que o artigo aparecia pela primeira vez, na seguinte ordem: PubMed, LILACS, SciELO, Capes.

A seleção dos estudos foi realizada de forma independente por dois pesquisadores com experiência em revisões, a partir da análise dos títulos, resumos e textos completos das publicações. As divergências foram resolvidas por consenso e, na sua impossibilidade, por um terceiro pesquisador com experiência no tema. Também foram consultadas as referências bibliográficas dos documentos selecionados para identificar e incluir outros estudos não capturados na busca eletrônica. Quando os textos completos selecionados não puderam ser obtidos em páginas da internet, foi realizado contato com o autor para solicitar a disponibilização do estudo.

Dos estudos incluídos, foram coletadas e registradas, em um banco de dados para esta pesquisa, informações sobre: objetivo, população, cenário de estudo, desenho do estudo, indicadores utilizados, método aplicado, ajustes realizados, fatores associados à qualidade (relacionados ao paciente e ao hospital), abordagem da qualidade (estrutura, processo, resultado), dimensão da qualidade avaliada (incluindo aceitabilidade, respeito ao direito das pessoas, continuidade, segurança, efetividade, eficiência) e principais resultados.

Foram consideradas ajustadas as taxas que levaram em conta, pelo menos, a idade dos pacientes ou, no caso de populações restritas em relação a essa variável, pelo menos, um outro fator. Para classificação da técnica estatística empregada nos casos em que mais de uma delas foi utilizada, considerou-se aquela mais complexa; no caso de estudos que realizaram análises estatísticas e também análises qualitativas, optou-se por sua classificação em ambos os grupos.

Para classificação dos indicadores segundo abordagem da qualidade (estrutura, processo, resultado), os autores consideraram os critérios definidos por Donabedian 28. Para classificação da dimensão da qualidade avaliada nos estudos, foram consideradas aquelas definidas pelo PRO-ADESS 26,27.

Não foram aplicados instrumentos para avaliação da qualidade metodológica dos estudos como critério de inclusão na análise, uma vez que, nesta revisão, não se pretendia sintetizar os resultados encontrados, mas sim analisar o desenvolvimento de estudos de avaliação no Brasil, inclusive apontando aspectos referentes à metodologia utilizada. Nesse sentido, não foram aplicadas medidas de sumarização dos resultados.

\section{Resultados}

Foram capturados, na busca eletrônica, 2.169 documentos. Desse total, 1.844 foram excluídos pela análise do título, 244 após leitura e análise do resumo e 22 após leitura e análise do texto completo. A partir das referências bibliográficas dos 59 documentos selecionados, identificaram- 
se mais 23 outros documentos para leitura do resumo e aplicação dos critérios de inclusão. Após a leitura de seus textos completos, 20 desses documentos foram excluídos, e 3 foram incluídos, somando o total de 62 documentos incluídos neste estudo (Tabelas 1 e 2; Figura 1).

Na sua maioria, os documentos excluídos tratavam de estudos clínicos, avaliação de técnicas ou comparação de tratamentos, avaliação de tecnologias ou estudos descritivos da morbidade hospitalar. Além desses, somam-se, como importantes motivos de exclusão, entre os documentos avaliados com base na leitura do texto completo, estudos sobre métodos de avaliação de qualidade ou estudos sobre gestão em saúde.

O documento mais antigo selecionado foi publicado no ano de 1991. No período de 1991 a 2003, foram publicados 1 ou 2 documentos por ano, somando 9 documentos no período. Em 2004, foram 5 documentos publicados sobre o assunto; a partir de então, observou-se maior número de publicações por ano, totalizando 48 documentos publicados entre 2004 e 2011.

Destacaram-se, entre os documentos selecionados: 11 artigos de Cadernos de Saúde Pública, 9 dissertações de mestrado, 8 artigos da Revista de Saúde Pública, 5 dos Arquivos Brasileiros de Cardiologia, 3 da Revista Latino-Americana de Enfermagem e 3 teses de doutorado. Outras 21 revistas tiveram artigos selecionados para esta revisão; as revistas nacionais foram o mais frequente meio de divulgação das análises, apesar de também serem observadas publicações em 10 periódicos internacionais.

Entre os 62 documentos selecionados, observaram-se 14 documentos publicados em multiplicidade, ou seja, reportando aspectos dos mesmos estudos em mais de um artigo ou em uma tese e um ou mais artigos. Em 8 estudos, observaram-se 2 documentos publicados; e, em 3 estudos, 3 documentos publicados. Com isso, as análises apresentadas nesta revisão sobre avaliações de qualidade realizadas no Brasil referem-se a um total de 62 publicações e 48 estudos diferentes (Figura 1; Tabela 2). Destaca-se que, entre as 9 teses e dissertações selecionadas, apenas 3 possuíam publicação em forma de artigo referente ao mesmo estudo.

Os desenhos dos 48 estudos analisados são majoritariamente observacionais, de corte transversal (42), tendo sido observados 3 estudos qualitativos, 2 estudos do tipo caso-controle e 1 estudo de coorte para o qual foram publicados 2 documentos, um deles reportando resultados da coorte, e o outro apresentando dados oriundos de caso-controle aninhado.

A principal fonte de informação utilizada foi o Sistema de Informações Hospitalares do SUS
(SIH/SUS), aplicada em 18 estudos. Sistemas de informação locais foram descritos como fontes de informações em 14 estudos, e os prontuários de pacientes, em 12. Já a coleta de dados primários, por entrevista, questionário ou observação direta, foi reportada em 15 estudos. Utilizaram combinações entre tais fontes 12 estudos.

A maioria dos estudos $(n=22)$ utilizou apenas 1 indicador. Em 18 estudos, foram utilizados 2 ou 3 indicadores, e, em 5, foram aplicados de 4 a 6 indicadores. Observaram-se 3 estudos que não calcularam indicadores e se dedicaram a análises qualitativas.

Quanto à abordagem da qualidade observada nos estudos, 21 estudos analisaram aspectos relacionados ao processo e resultado, 12 estudos trataram de estrutura, processo e resultado, 7 estudos apenas de processo, 6 estudos de resultado e 2 estudos de estrutura e processo. Em relação às dimensões de avaliação exploradas, 32 estudos abordaram indicadores de efetividade, 16 de adequação, 12 de segurança, 12 de eficiência, 10 de respeito aos direitos das pessoas, 6 de acesso, 4 de continuidade e 1 de aceitabilidade (Tabela 3).

Entre os indicadores aplicados, destaca-se a taxa de mortalidade hospitalar, medida da dimensão efetividade calculada em 29 estudos, sendo, em 18 deles, referente à mortalidade específica por causa selecionada e, em 11 deles, à mortalidade total. A razão entre óbitos observados e esperados foi calculada em 6 estudos, e os óbitos por causas evitáveis foram estimados em 3 estudos. Além de constituir o indicador mais frequentemente utilizado como medida de desempenho dos serviços, a taxa de mortalidade foi aplicada exclusivamente em 10 estudos. Já a taxa de readmissão, aplicada como medida da efetividade dos serviços hospitalares prestados, foi calculada em apenas 1 estudo, em que também aplicou-se a taxa de mortalidade. A efetividade do cuidado também foi explorada em dois estudos qualitativos que não calcularam qualquer taxa e em 1 estudo que abordou a efetividade como fator de avaliação para composição da taxa de satisfação (Tabela 4).

Dos 16 estudos que analisaram o desempenho a partir da dimensão adequação, 11 também mediram efetividade a partir do cálculo da taxa de mortalidade, e 2 aplicaram exclusivamente a taxa de adequação como medida de desfecho. Os principais temas explorados no que diz respeito à adequação relacionam-se aos cuidados a pacientes com AVC, pacientes cardíacos, pacientes em trabalho de parto ou pacientes elegíveis para protocolos específicos, à realização de parto cesáreo e ao cuidado de enfermagem. Além da taxa de adequação ao cuidado, medida em 14 estudos, 1 estudo abordou a dimensão adequação a 
Tabela 1

Estratégias de busca segundo fonte de pesquisa e resultado obtido

\begin{tabular}{|c|c|c|c|c|c|}
\hline \multirow{2}{*}{$\begin{array}{l}\text { Fontes de } \\
\text { pesquisa }\end{array}$} & \multirow[t]{2}{*}{ Regras de busca } & \multicolumn{4}{|c|}{ Resultados (n) } \\
\hline & & Total & $\begin{array}{l}\text { Seleção por } \\
\text { títulos }\end{array}$ & $\begin{array}{l}\text { Seleção por } \\
\text { resumos }\end{array}$ & $\begin{array}{c}\text { Seleção } \\
\text { final }\end{array}$ \\
\hline \multirow[t]{16}{*}{ PubMed } & ("hospitals" [MesH Terms] OR hospital [Text Word] OR hospitalization) & 1.299 & 208 & 37 & 31 \\
\hline & AND ("Brazil" [MeSH Terms] OR Brazil [Text Word]) & & & & \\
\hline & AND (("outcome assessment (health care)" [Mesh Terms]) OR ("process & & & & \\
\hline & assessment (health care)" [Mesh Terms]) OR ("quality of health care" [Mesh Major & & & & \\
\hline & Topic]) OR ("quality assurance, health care" [Mesh Terms) OR ("Health Care & & & & \\
\hline & Evaluation Mechanisms" [Mesh Major Topic]) OR ("quality indicators, health care" & & & & \\
\hline & [MeSH Terms]) OR ("hospital mortality" [MeSH Terms]) OR ("length of stay" [MeSH & & & & \\
\hline & Terms]) OR ("efficiency, organizational" [MeSH Terms]) OR ("patient safety" [MeSH & & & & \\
\hline & Terms]) OR ("medical errors" [MeSH Terms]) OR ("patient acceptance of health & & & & \\
\hline & care" [MeSH Terms]) OR ("patient-centered care" [MeSH Terms]) OR ("quality of & & & & \\
\hline & health care" [Text Word]) OR ("healthcare quality indicator" [Text Word])) & & & & \\
\hline & Limites: Humans, Meta-Analysis, Randomized Controlled Trial, Review, Classical & & & & \\
\hline & Article, Comparative Study, Evaluation Studies, Government Publications, Journal & & & & \\
\hline & Article, Multicenter Study, Scientific Integrity Review, Technical Report, Twin Study, & & & & \\
\hline & Validation Studies. & & & & \\
\hline & Data de publicação: de 1ø/Jan/1990 até 31/Dez/2011 & & & & \\
\hline \multirow[t]{8}{*}{ LILACS } & Hospitais OR hospital OR hospitalização [Palavras] AND Brasil [Palavras] AND & 205 & 35 & 19 & 12 \\
\hline & "Garantia da Qualidade dos Cuidados de Saúde" OR "Avaliação de Resultados & & & & \\
\hline & (Cuidados de Saúde)" OR "Avaliação de Processos (Cuidados de Saúde)" & & & & \\
\hline & OR "Mecanismos de Avaliação em Cuidados de Saúde" OR "Indicadores de & & & & \\
\hline & Qualidade em Assistência à Saúde" OR "Mortalidade Hospitalar" OR "Tempo de & & & & \\
\hline & Permanência" OR "Eficiência Organizacional" OR "Erros Médicos" OR "Aceitação & & & & \\
\hline & pelo Paciente de Cuidados de Saúde" OR "Cuidado Centrado no Paciente" OR & & & & \\
\hline & "segurança do paciente" OR "Qualidade do Cuidado em Saúde" [Palavras] & & & & \\
\hline \multirow[t]{9}{*}{ SciELO } & Hospitais OR hospital OR hospitalização [Todos os índices] AND Brasil [Todos os & 73 & 23 & 11 & 7 \\
\hline & índices] AND "Garantia da Qualidade dos Cuidados de Saúde" OR "Avaliação & & & & \\
\hline & de Resultados (Cuidados de Saúde)" OR "Avaliação de Processos (Cuidados de & & & & \\
\hline & Saúde)" OR "Mecanismos de Avaliação em Cuidados de Saúde" OR "Indicadores & & & & \\
\hline & de Qualidade em Assistência à Saúde" OR "Mortalidade Hospitalar" OR "Tempo & & & & \\
\hline & de Permanência" OR "Eficiência Organizacional" OR "Erros Médicos" OR & & & & \\
\hline & "Aceitação pelo Paciente de Cuidados de Saúde" OR "Cuidado Centrado no & & & & \\
\hline & Paciente" OR "segurança do paciente" OR "Qualidade do Cuidado em Saúde" & & & & \\
\hline & [Todos os índices] & & & & \\
\hline \multirow[t]{7}{*}{ Capes } & Total busca & 592 & 59 & 14 & 9 \\
\hline & Avaliação de qualidade de serviços de saúde, hospital, indicadores & 154 & & & \\
\hline & Efetividade, hospital, indicadores & 184 & & & \\
\hline & Erros médicos, hospital, indicadores & 33 & & & \\
\hline & Segurança do paciente, hospital, indicadores & 103 & & & \\
\hline & Cuidado centrado no paciente, hospital, indicadores & 33 & & & \\
\hline & Aceitação pelo paciente, hospital, indicadores & 85 & & & \\
\hline Referências & Busca manual nas referências dos documentos selecionados & & & 23 & 3 \\
\hline Total & & 2.169 & 325 & 104 & 62 \\
\hline
\end{tabular}

Capes: Coordenação de Aperfeiçoamento de Pessoal de Nível Superior; LILACS: Literatura Latino-Americana e do Caribe em Ciências da Saúde; SciELO: Scientific Electronic Library Online. 
Tabela 2

Principais características dos estudos selecionados.

\begin{tabular}{|c|c|c|c|c|c|c|}
\hline Documento & Estudo & Referência & $\begin{array}{l}\text { Ano(s) dos } \\
\text { dados }\end{array}$ & População e cenário de estudo & $\begin{array}{l}\text { Fonte(s) de } \\
\text { informação }\end{array}$ & $\begin{array}{c}\text { Desenho do } \\
\text { estudo }\end{array}$ \\
\hline 1 & 1 & Gomes 47 & 2005 & $\begin{array}{c}\text { Adultos internados em clínica médica e } \\
\text { cirúrgica, em hospitais do Rio Grande } \\
\text { do Sul }\end{array}$ & $\mathrm{SIH} / \mathrm{SUS}$ & Transversal \\
\hline 2 & 1 & Gomes et al. 48 & 2005 & $\begin{array}{c}\text { Adultos internados em clínica médica e } \\
\text { cirúrgica, em hospitais do Rio Grande } \\
\text { do Sul }\end{array}$ & $\mathrm{SIH} / \mathrm{SUS}$ & Transversal \\
\hline 3 & 1 & Gomes et al. 49 & 2005 & $\begin{array}{c}\text { Adultos internados em clínica médica e } \\
\text { cirúrgica, em hospitais do Rio Grande } \\
\text { do Sul }\end{array}$ & $\mathrm{SIH} / \mathrm{SUS}$ & Transversal \\
\hline 4 & 2 & lucif Jr. et al. 60 & 1998-1999 & $\begin{array}{c}\text { Pacientes com 50+ anos internados } \\
\text { com doenças dos aparelhos respiratório } \\
\text { e circulatório em hospitais de Ribeirão } \\
\text { Preto (São Paulo) }\end{array}$ & $\begin{array}{l}\text { Sistema de } \\
\text { informações local }\end{array}$ & Transversal \\
\hline 5 & 3 & Martins et al. 51 & 1996-1998 & $\begin{array}{c}\text { Pacientes com } 50+\text { anos internados } \\
\text { com doenças dos aparelhos respiratório } \\
\text { e circulatório em hospitais de Ribeirão } \\
\text { Preto (São Paulo) }\end{array}$ & $\begin{array}{l}\text { Sistema de } \\
\text { informações local }\end{array}$ & Transversal \\
\hline 6 & 4 & Mesquita et al. 35 & 2005-2007 & $\begin{array}{l}\text { Pacientes internados para cirurgia } \\
\text { de revascularização do miocárdio no } \\
\text { Hospital Pró-Cardíaco, Rio de Janeiro }\end{array}$ & $\begin{array}{l}\text { Sistema de } \\
\text { informações local }\end{array}$ & Transversal \\
\hline 7 & 4 & Rocha et al. 34 & 2006-2007 & $\begin{array}{c}\text { Pacientes internados com IAM no } \\
\text { Hospital Pró-Cardíaco, Rio de Janeiro }\end{array}$ & $\begin{array}{l}\text { Sistema de } \\
\text { informações local }\end{array}$ & Transversal \\
\hline 8 & 5 & Rolim 52 & 2006-2007 & $\begin{array}{c}\text { Adultos internados por AVC em } \\
\text { hospitais do Brasil }\end{array}$ & SIH/SUS & Transversal \\
\hline 9 & 5 & Rolim \& Martins 53 & $2006-2007$ & $\begin{array}{l}\text { Adultos internados por AVC a menos de } \\
7 \text { dias em hospitais do Brasil }\end{array}$ & $\mathrm{SIH} / \mathrm{SUS}$ & Transversal \\
\hline 10 & 6 & Daud-Gallotti et al. 54 & 1996-1999 & $\begin{array}{c}\text { Pacientes com AVC admitidos na } \\
\text { emergência de um hospital universitário } \\
\text { em São Paulo }\end{array}$ & Prontuários & $\begin{array}{l}\text { Caso- } \\
\text { controle }\end{array}$ \\
\hline 11 & 7 & Noronha 55 & 1996-1998 & $\begin{array}{c}\text { Pacientes internados para cirurgia de } \\
\text { revascularização do miocárdio em } \\
\text { hospitais do Brasil }\end{array}$ & $\mathrm{SIH} / \mathrm{SUS}$ & Transversal \\
\hline 12 & 7 & Noronha et al. 56 & 1996-1998 & $\begin{array}{c}\text { Pacientes internados para cirurgia de } \\
\text { revascularização do miocárdio em } \\
\text { hospitais do Brasil }\end{array}$ & $\mathrm{SIH} / \mathrm{SUS}$ & Transversal \\
\hline 13 & 7 & Noronha et al. 57 & 1996-1998 & $\begin{array}{l}\text { Pacientes internados para cirurgia de } \\
\text { revascularização do miocárdio em } \\
\text { hospitais do Brasil com mais de } 150 \\
\text { procedimentos no período }\end{array}$ & $\mathrm{SIH} / \mathrm{SUS}$ & Transversal \\
\hline 14 & 8 & Paiva 58 & 2005 & $\begin{array}{l}\text { Pacientes que se internaram ao menos } \\
\text { por } 4 \text { dias em clínica médica de um } \\
\text { hospital do interior de São Paulo }\end{array}$ & $\begin{array}{c}\text { Coleta de dados } \\
\text { primários }\end{array}$ & Qualitativo \\
\hline 15 & 8 & Paiva et al. 59 & 2005 & $\begin{array}{l}\text { Pacientes que se internaram ao menos } \\
\text { por } 4 \text { dias em clínica médica de um } \\
\text { hospital do interior de São Paulo }\end{array}$ & $\begin{array}{l}\text { Coleta de dados } \\
\text { primários }\end{array}$ & Qualitativo \\
\hline 16 & 9 & Godoy et al. 33 & 1999-2003 & $\begin{array}{c}\text { Pacientes submetidos a angioplastias } \\
\text { coronárias financiadas pelo SUS no Rio } \\
\text { de Janeiro }\end{array}$ & $\mathrm{SIH} / \mathrm{SUS}$ & Transversal \\
\hline
\end{tabular}

(continua) 
Tabela 2 (continuação)

\begin{tabular}{|c|c|c|c|c|c|c|}
\hline Documento & Estudo & Referência & $\begin{array}{l}\text { Ano(s) dos } \\
\text { dados }\end{array}$ & População e cenário de estudo & $\begin{array}{l}\text { Fonte(s) de } \\
\text { informação }\end{array}$ & $\begin{array}{l}\text { Desenho do } \\
\text { estudo }\end{array}$ \\
\hline 17 & 9 & Oliveira et al. 60 & $1999-2003$ & $\begin{array}{l}\text { Pacientes internados para cirurgia de } \\
\text { revascularização do miocárdio em } 4 \\
\text { hospitais no Rio de Janeiro }\end{array}$ & $\begin{array}{l}\text { SIH/SUS, } \\
\text { prontuários e SIM }\end{array}$ & Transversal \\
\hline 18 & 10 & Kelles et al. 61 & 2004-2007 & $\begin{array}{c}\text { Pacientes internados para cirurgia } \\
\text { bariátrica aberta em Belo Horizonte } \\
\text { (Minas Gerais) }\end{array}$ & $\begin{array}{l}\text { Sistema de } \\
\text { informações local e } \\
\text { prontuários }\end{array}$ & Transversal \\
\hline 19 & 11 & Mendes et al. 62 & 2003 & $\begin{array}{l}\text { Pacientes internados em } 3 \text { hospitais de } \\
\text { ensino no Rio de Janeiro }\end{array}$ & Prontuários & Transversal \\
\hline 20 & 11 & Martins et al. 63 & 2003 & $\begin{array}{l}\text { Pacientes internados em } 3 \text { hospitais de } \\
\text { ensino no Rio de Janeiro }\end{array}$ & Prontuários & Transversal \\
\hline 21 & 12 & Moura et al. 64 & 2007 & $\begin{array}{c}\text { Pacientes com } 18+\text { anos internados em } \\
\text { hospital público na Bahia }\end{array}$ & $\begin{array}{l}\text { SIH/SUS e sistema } \\
\text { de informações } \\
\text { local }\end{array}$ & Transversal \\
\hline 22 & 12 & Moura et al. 65 & 2007 & $\begin{array}{l}\text { Pacientes internados em UTI no Hospital } \\
\text { Geral de Vitória da Conquista, Bahia }\end{array}$ & $\begin{array}{l}\text { SIH/SUS e sistema } \\
\text { de informações } \\
\text { local }\end{array}$ & Transversal \\
\hline 23 & 13 & Nascimento et al. 66 & 2006 & $\begin{array}{c}\text { Pacientes a partir de } 18 \text { anos que } \\
\text { sofreram evento adverso durante } \\
\text { internação em hospital privado em São } \\
\text { Paulo }\end{array}$ & $\begin{array}{l}\text { Sistema de } \\
\text { informações local }\end{array}$ & Transversal \\
\hline 24 & 14 & Rosa et al. 67 & 1994 & $\begin{array}{c}\text { Mulheres internadas em } 4 \text { maternidades } \\
\text { da rede SUS na Região Metropolitana } \\
\text { do Rio de Janeiro }\end{array}$ & $\begin{array}{l}\text { SIH/SUS, SIM, } \\
\text { sistema de } \\
\text { informações local, } \\
\text { coleta de dados } \\
\text { primários }\end{array}$ & Qualitativo \\
\hline 25 & 15 & Chiavone et al. 68 & $2002-2003$ & $\begin{array}{l}\text { Pacientes internados em UTI pós- } \\
\text { cirurgia emergencial no Hospital Santa } \\
\text { Casa de Misericórdia de São Paulo }\end{array}$ & $\begin{array}{l}\text { Coleta de dados } \\
\text { primários }\end{array}$ & Transversal \\
\hline 26 & 16 & Amaral et al. 69 & 1999 & $\begin{array}{c}\text { Pacientes a partir de } 60 \text { anos internados } \\
\text { em } 4 \text { hospitais da AP2.2, no Rio de } \\
\text { Janeiro }\end{array}$ & $\mathrm{SIH} / \mathrm{SUS}$ & Transversal \\
\hline 27 & 17 & Dias 70 & 2007 & $\begin{array}{l}\text { Adultos internados em clínica médica e } \\
\text { cirúrgica em hospitais do Brasil }\end{array}$ & SIH/SUS & Transversal \\
\hline 28 & 18 & Escosteguy et al. 71 & 1997 & $\begin{array}{l}\text { Pacientes internados com diagnóstico } \\
\text { de IAM em hospitais do Rio de Janeiro }\end{array}$ & SIH/SUS & Transversal \\
\hline 29 & 19 & Piegas et al. 30 & $2005-2007$ & $\begin{array}{c}\text { Pacientes internados para intervenção } \\
\text { coronariana percutânea em hospitais } \\
\text { do Brasil }\end{array}$ & $\mathrm{SIH} / \mathrm{SUS}$ & Transversal \\
\hline 30 & 19 & Piegas \& Haddad 29 & $2005-2008$ & $\begin{array}{c}\text { Pacientes internados para intervenção } \\
\text { coronariana percutânea em hospitais } \\
\text { do Brasil }\end{array}$ & $\mathrm{SIH} / \mathrm{SUS}$ & Transversal \\
\hline 31 & 20 & Borges et al. 72 & 2006 & $\begin{array}{c}\text { Pacientes submetidos à cirurgia cardíaca } \\
\text { eletiva no SUS da Santa Casa de } \\
\text { Misericórdia de Marília (São Paulo) }\end{array}$ & $\begin{array}{l}\text { Coleta de dados } \\
\text { primários }\end{array}$ & Transversal \\
\hline 32 & 21 & Cesconetto et al. 73 & 2003 & $\begin{array}{c}\text { Pacientes internados em hospitais gerais } \\
\text { de Santa Catarina }\end{array}$ & $\mathrm{SIH} / \mathrm{SUS}$ & Transversal \\
\hline 33 & 22 & Costa et al. 74 & 2005 & $\begin{array}{l}\text { Pacientes internados em clínica médica } \\
\text { ou cirúrgica de um hospital público e de } \\
\text { um hospital privado na Bahia }\end{array}$ & $\begin{array}{c}\text { Coleta de dados } \\
\text { primários }\end{array}$ & Transversal \\
\hline 34 & 23 & Gouvêa et al. 31 & 1992-1995 & $\begin{array}{l}\text { Pacientes internados em hospitais no } \\
\text { Rio de Janeiro }\end{array}$ & $\mathrm{SIH} / \mathrm{SUS}$ & Transversal \\
\hline
\end{tabular}

(continua) 
Tabela 2 (continuação)

\begin{tabular}{|c|c|c|c|c|c|c|}
\hline Documento & Estudo & Referência & $\begin{array}{l}\text { Ano(s) dos } \\
\text { dados }\end{array}$ & População e cenário de estudo & $\begin{array}{l}\text { Fonte(s) de } \\
\text { informação }\end{array}$ & $\begin{array}{l}\text { Desenho do } \\
\text { estudo }\end{array}$ \\
\hline 35 & 24 & Gonçalves et al. 75 & 2000 & $\begin{array}{c}\text { Pacientes internados em clínicas } \\
\text { médicas de hospitais de capitais do } \\
\text { Brasil }\end{array}$ & $\mathrm{SIH} / \mathrm{SUS}$ & Transversal \\
\hline 36 & 25 & Kotaka et al. 76 & 1994 & $\begin{array}{l}\text { Pacientes internados em hospitais que } \\
\text { participam de programa de qualidade } \\
\text { em São Paulo }\end{array}$ & $\begin{array}{l}\text { Coleta de dados } \\
\text { primários }\end{array}$ & Qualitativo \\
\hline 37 & 26 & Lansky et al. 77 & 1999 & $\begin{array}{l}\text { Coorte de nascidos em hospitais de } \\
\text { Belo Horizonte (Minas Gerais) e suas } \\
\text { mães }\end{array}$ & $\begin{array}{l}\text { Prontuários, sistema } \\
\text { de informações } \\
\text { local, SINASC e SIM }\end{array}$ & Coorte \\
\hline 38 & 26 & Lansky et al. 78 & 1999 & $\begin{array}{l}\text { Coorte de nascidos em hospitais de } \\
\text { Belo Horizonte (Minas Gerais) e suas } \\
\text { mães }\end{array}$ & $\begin{array}{l}\text { Prontuários, sistema } \\
\text { de informações } \\
\text { local, SINASC e SIM }\end{array}$ & $\begin{array}{l}\text { Caso- } \\
\text { controle }\end{array}$ \\
\hline 39 & 26 & Lansky et al. 79 & 1999 & $\begin{array}{l}\text { Coorte de nascidos em hospitais de } \\
\text { Belo Horizonte (Minas Gerais) e suas } \\
\text { mães }\end{array}$ & $\begin{array}{l}\text { Prontuários, sistema } \\
\text { de informações } \\
\text { local, SINASC e SIM }\end{array}$ & Coorte \\
\hline 40 & 27 & Oliveira et al. 80 & 1999-2001 & $\begin{array}{c}\text { Amostra de parturientes internadas em } \\
\text { hospitais do SUS no Rio de Janeiro }\end{array}$ & $\begin{array}{l}\text { Prontuários e coleta } \\
\text { de dados primários }\end{array}$ & Transversal \\
\hline 41 & 28 & Louro et al. 81 & $2002-2003$ & $\begin{array}{c}\text { Pacientes internados em hospital } \\
\text { universitário de Maringá (Paraná), em } \\
\text { antibioticoterapia }\end{array}$ & $\begin{array}{l}\text { Prontuários e coleta } \\
\text { de dados primários }\end{array}$ & Transversal \\
\hline 42 & 29 & Masella et al. 82 & $2000-2001$ & $\begin{array}{c}\text { Pacientes vítimas de trauma no Hospital } \\
\text { das Clínicas de Ribeirão Preto (São } \\
\text { Paulo) }\end{array}$ & SIM e prontuários & Transversal \\
\hline 43 & 30 & Matos et al. 83 & $1999-2001$ & $\begin{array}{l}\text { Pacientes internados em hospitais da } \\
\text { Região Metropolitana do Rio de Janeiro } \\
\text { em uso de albumina humana }\end{array}$ & $\mathrm{SIH} / \mathrm{SUS}$ & Transversal \\
\hline 44 & 31 & Passarelli et al. 84 & $2002-2004$ & $\begin{array}{l}\text { Pacientes idosos internados no Hospital } \\
\text { Municipal Central de Santo André (São } \\
\text { Paulo) }\end{array}$ & $\begin{array}{l}\text { Coleta de dados } \\
\text { primários }\end{array}$ & Transversal \\
\hline 45 & 32 & Ricci et al. 85 & $2007-2008$ & $\begin{array}{l}\text { Pacientes atendidos no Hospital Escola } \\
\text { de São Carlos }\end{array}$ & $\begin{array}{l}\text { Coleta de dados } \\
\text { primários }\end{array}$ & Transversal \\
\hline 46 & 33 & Schramm et al. 86 & 1997 & $\begin{array}{c}\text { Pacientes com internação obstétrica em } \\
\text { hospitais do Rio de Janeiro }\end{array}$ & $\mathrm{SIH} / \mathrm{SUS}$ & Transversal \\
\hline 47 & 34 & Silva et al. 87 & 2002-2007 & $\begin{array}{c}\text { Pacientes internados na clínica médica } \\
\text { de hospital em Goiás }\end{array}$ & $\begin{array}{l}\text { Sistema de } \\
\text { informações local }\end{array}$ & Transversal \\
\hline 48 & 35 & Carneiro et al. 88 & 2005-2009 & $\begin{array}{c}\text { Pacientes internados na clínica cirúrgica, } \\
\text { com eventos adversos, em hospital de } \\
\text { Goiânia (Goiás) }\end{array}$ & $\begin{array}{l}\text { Sistema de } \\
\text { informações local }\end{array}$ & Transversal \\
\hline 49 & 35 & Souza et al. 89 & $2005-2009$ & $\begin{array}{l}\text { Pacientes que passaram pelo centro } \\
\text { cirúrgico, com eventos adversos, em } \\
\text { hospital de Goiânia (Goiás) }\end{array}$ & $\begin{array}{l}\text { Sistema de } \\
\text { informações local }\end{array}$ & Transversal \\
\hline 50 & 36 & Cruz 90 & 2007 & $\begin{array}{l}\text { Pacientes internados no Hospital } \\
\text { Adventista de São Paulo }\end{array}$ & $\begin{array}{l}\text { Coleta de dados } \\
\text { primários }\end{array}$ & Transversal \\
\hline 51 & 37 & Marinho 91 & 1995 & Hospitais de grande porte de São Paulo & $\begin{array}{l}\text { Sistema de } \\
\text { informações local }\end{array}$ & Transversal \\
\hline 52 & 38 & Zucchi 92 & 1995 & Hospitais de grande porte de São Paulo & $\begin{array}{l}\text { Sistema de } \\
\text { informações local }\end{array}$ & Transversal \\
\hline 53 & 39 & Martins 93 & 1986 & $\begin{array}{c}\text { Pacientes internados em } 36 \text { hospitais do } \\
\text { Rio de Janeiro }\end{array}$ & $\begin{array}{l}\text { Sistema de } \\
\text { informações local }\end{array}$ & Transversal \\
\hline
\end{tabular}

(continua) 


\begin{tabular}{|c|c|c|c|c|c|c|}
\hline Documento & Estudo & Referência & $\begin{array}{l}\text { Ano(s) dos } \\
\text { dados }\end{array}$ & População e cenário de estudo & $\begin{array}{l}\text { Fonte(s) de } \\
\text { informação }\end{array}$ & $\begin{array}{l}\text { Desenho do } \\
\text { estudo }\end{array}$ \\
\hline 54 & 40 & Costa 94 & 1993 & $\begin{array}{c}\text { Pacientes menores de } 5 \text { anos internados } \\
\text { por IRA na clínica médica do Hospital } \\
\text { Municipal Jesus }\end{array}$ & Prontuários & Transversal \\
\hline 55 & 41 & Gonçalves 95 & 1991 e 2000 & Idosos internados no Brasil com IAM & $\begin{array}{l}\text { SIH/SUS, SIM e } \\
\text { AMS }\end{array}$ & Transversal \\
\hline 56 & 42 & Golovattei 96 & 2008-2009 & $\begin{array}{l}\text { Hospitais da Região Metropolitana de } \\
\text { São Paulo }\end{array}$ & $\begin{array}{l}\text { Sistema de } \\
\text { informações local }\end{array}$ & Transversal \\
\hline 57 & 43 & Alves 97 & 1997 & $\begin{array}{c}\text { Maternidades com mais de } 100 \text { partos } \\
\text { em 1996, em São Luís (Maranhão) }\end{array}$ & $\begin{array}{c}\text { Coleta de dados } \\
\text { primários, } \\
\text { prontuário e } \\
\text { sistema de } \\
\text { informações local }\end{array}$ & Transversal \\
\hline 58 & 44 & Costa 98 & 2009-2010 & $\begin{array}{l}\text { Pacientes entre 0-28 dias de vida, } \\
\text { internados na UTI neonatal de hospital } \\
\text { universitário no Rio Grande do Sul }\end{array}$ & $\begin{array}{l}\text { Coleta de dados } \\
\text { primários e } \\
\text { prontuário }\end{array}$ & Transversal \\
\hline 59 & 45 & Haddad 99 & $1988-2003$ & $\begin{array}{c}\text { Pacientes submetidos à cirurgia } \\
\text { de revascularização do miocárdio } \\
\text { do Instituto Dante Pazzanese de } \\
\text { Cardiologia, São Paulo }\end{array}$ & $\begin{array}{l}\text { Sistema de } \\
\text { informações local }\end{array}$ & Transversal \\
\hline 60 & 46 & Santos et al. 100 & $\begin{array}{c}\text { Não } \\
\text { descreve }\end{array}$ & $\begin{array}{c}\text { Pacientes internados em seis hospitais } \\
\text { de João Pessoa (Paraíba) conveniados } \\
\text { ao SUS }\end{array}$ & $\begin{array}{l}\text { Coleta de dados } \\
\text { primários }\end{array}$ & Transversal \\
\hline 61 & 47 & d'Orsi et al. 101 & 1998-1999 & $\begin{array}{l}\text { Mulheres em uma maternidade pública } \\
\text { e mulheres em uma maternidade } \\
\text { conveniada no Rio de Janeiro }\end{array}$ & $\begin{array}{l}\text { Prontuário e coleta } \\
\text { de dados primários }\end{array}$ & $\begin{array}{l}\text { Caso- } \\
\text { controle }\end{array}$ \\
\hline 62 & 48 & Guerra et al. 102 & 1999-2002 & $\begin{array}{l}\text { Hospitais das capitais brasileiras, que } \\
\text { realizaram pelo menos } 200 \text { internações, } \\
\text { pelo SUS, de pacientes idosos com } \\
\text { procedimento principal "Atendimento a } \\
\text { pacientes sob cuidados prolongados" }\end{array}$ & $\mathrm{SIH} / \mathrm{SUS}$ & Transversal \\
\hline
\end{tabular}

AMS: Pesquisa de Assistência Médico-Sanitária; AVC: acidente vascular cerebral; IAM: infarto agudo do miocárdio; IRA: infecções respiratórias agudas; SIH/ SUS: Sistema de Informações Hospitalares do SUS; SIM: Sistema de Informações sobre Mortalidade; SINASC: Sistema de Informações sobre Nascidos Vivos; SUS: Sistema Único de Saúde; UTI: unidade de terapia intensiva.

partir de análise qualitativa sem cálculo de taxas, e 1 estudo abordou a adequação como fator de avaliação para composição da taxa de satisfação (Tabela 4).

Sobre os 12 estudos que abordaram a dimensão segurança, a taxa de eventos adversos e a medida dos erros de medicação foram indicadores aplicados respectivamente em 11 e em 4 estudos, tendo sido utilizadas exclusivamente como medidas de desfecho em 2 estudos cada uma (Tabela 4).

A eficiência, enquanto indicador sintético, foi aplicada em 3 estudos; os custos de internação, em 3 estudos; e a medida do tempo de permanência foi aplicada em 7 estudos entre os 12 que exploraram a dimensão eficiência. Um estudo explorou essa dimensão a partir de análise qualitativa, sem calcular taxa (Tabela 4).

Entre os 8 estudos que exploraram a dimensão respeito ao direito dos pacientes, incluem-se 3 estudos qualitativos que não calcularam taxas, e 5 estudos que aplicaram medidas sintéticas de satisfação (Tabela 4).

Dos 6 estudos que envolveram análise da dimensão acesso, 3 eram estudos qualitativos que não realizaram cálculo de indicadores, 2 medi- 


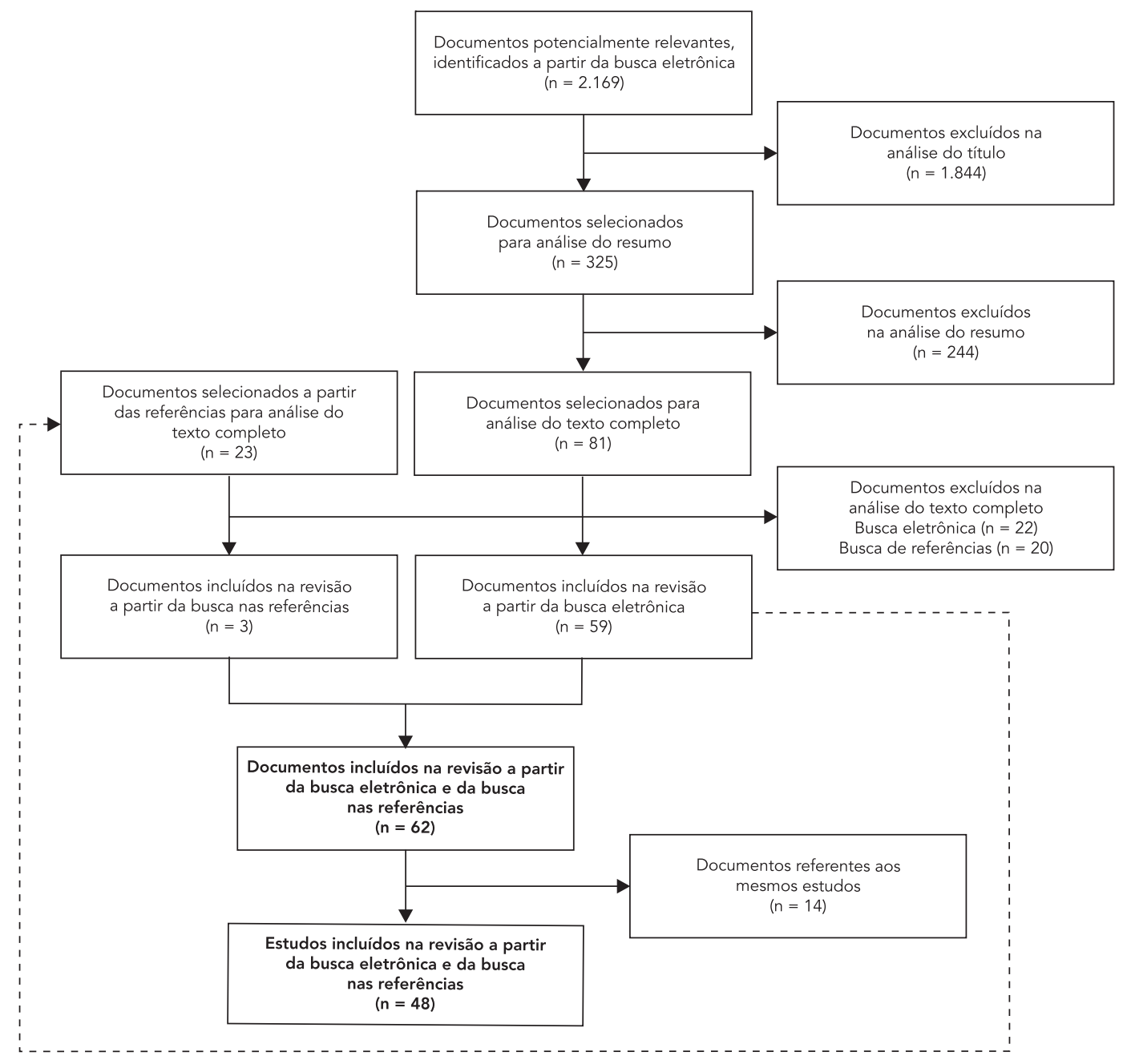

ram acesso a partir do tempo entre a indicação de procedimento ou de transferência entre setores/estabelecimentos e sua realização, e 1 incluiu aspectos do acesso como fatores de avaliação para composição da taxa de satisfação (Tabela 4).

As dimensões continuidade e aceitabilidade foram exploradas em estudos que aplicaram análises qualitativas e não calcularam taxas (Tabela 4).

Não houve estudos selecionados que utilizassem indicadores ou aplicassem análises qualitativas específicas sobre a dimensão equidade que, conforme o PRO-ADESS, perpassa todas as demais dimensões de avaliação. Apesar disso, em alguns estudos, a discussão dos resultados considerou aspectos relacionados a essa dimensão destacando sua importância para a análise das demais dimensões.

Também foram incluídos 14 estudos que, além das dimensões relacionadas ao desempenho dos serviços de saúde, analisaram ainda a dimensão recursos, relacionada à estrutura. Desses, 6 estudos utilizaram taxas de adequação da estrutura, 4 estudos incluíram aspectos da estrutura como fatores de avaliação para composição da taxa de satisfação, 2 estudos incluíram aspectos da estrutura como fatores de avaliação para composição da taxa sintética de eficiência, 
Número de estudos selecionados segundo abordagem e dimensão da qualidade, métodos de análise e ranqueamento de serviços.

\begin{tabular}{|c|c|c|}
\hline Categoria & $n(N=48)$ & $\%$ \\
\hline \multicolumn{3}{|l|}{ Abordagem da qualidade } \\
\hline Processo e resultado & 21 & 43,8 \\
\hline Estrutura, processo e resultado & 12 & 25,0 \\
\hline Processo & 7 & 14,6 \\
\hline Resultado & 6 & 12,5 \\
\hline Estrutura e processo & 2 & 4,2 \\
\hline \multicolumn{3}{|l|}{ Dimensão da qualidade * } \\
\hline Efetividade & 32 & 66,7 \\
\hline Adequação & 16 & 33,3 \\
\hline Eficiência & 12 & 25,0 \\
\hline Segurança & 12 & 25,0 \\
\hline Respeito ao direito das pessoas & 8 & 16,7 \\
\hline Acesso & 6 & 12,5 \\
\hline Continuidade & 3 & 6,3 \\
\hline Aceitabilidade & 1 & 2,1 \\
\hline Recursos (da estrutura) ** & 6 & 12,5 \\
\hline \multicolumn{3}{|l|}{ Métodos de análise * } \\
\hline Taxas brutas, sem ajustes & 16 & 33,3 \\
\hline Taxas brutas de mortalidade para comparar hospitais ou serviços entre si ou no tempo & 4 & 8,3 \\
\hline Taxas estratificadas ou ajustadas & 8 & 16,7 \\
\hline Regressão logística & 11 & 22,9 \\
\hline Regressão linear & 7 & 14,6 \\
\hline Análise multinível & 3 & 6,3 \\
\hline Análise envoltória de dados (DEA) & 3 & 6,3 \\
\hline Análise fatorial & 1 & 2,1 \\
\hline Análises qualitativas & 4 & 8,3 \\
\hline \multicolumn{3}{|l|}{ Ranqueamento de estabelecimentos ou serviços } \\
\hline Sim & 17 & 35,4 \\
\hline Não & 31 & 64,6 \\
\hline
\end{tabular}

* Nessa categoria de análise, os estudos puderam ser classificados em mais de uma resposta;

** Além das dimensões relacionadas ao desempenho dos serviços, alguns estudos analisaram também aspectos da dimensão "recursos", relacionada à estrutura.

e 2 estudos exploraram essa dimensão a partir de análise qualitativa, sem calcular taxas. A dimensão financiamento, relacionada também à estrutura, não foi aplicada nos estudos selecionados (Tabela 4).

O método predominantemente aplicado para analisar a qualidade dos serviços hospitalares a partir das medidas descritas foi o cálculo de taxas brutas (16 estudos). Taxas estratificadas ou ajustadas com análises bivariadas foram utilizadas em 8 estudos. Já a regressão logística foi aplicada em 1lestudos; a regressão linear, em 7 estudos; a análise envoltória de dados, em 3 estudos; a análise multinível, em outros 3 estudos, enquanto 1 estudo aplicou análise fatorial pelo método de componentes principais. Dos estudos que aplicaram regressões, a maioria também apresentou estatísticas descritivas em forma de taxas brutas ou estratificadas. Por fim, 4 estudos realizaram análises qualitativas, todos eles abrangendo aspectos relacionados à abordagem da estrutura, do processo e do resultado (Tabela 3).

Dos 16 estudos que utilizaram taxas brutas como medida do desfecho, a maioria mediu eventos adversos, erros de medicação, adequação de cuidados e satisfação. No entanto, em quatro estudos, foi calculada a taxa bruta de mortalidade 29,30,31,32,33,34,35, aplicada, em três deles, 
Tabela 4

Estudos selecionados segundo dimensões e indicadores aplicados para medir o desempenho dos serviços de saúde.

\begin{tabular}{|c|c|c|c|c|c|}
\hline $\begin{array}{l}\text { Macrodimensão } \\
\text { (Viacava et al. }{ }^{27} \text { ) }\end{array}$ & $\begin{array}{c}\text { Dimensão } \\
\text { (Viacava et } \\
\text { al. 27) }\end{array}$ & $\begin{array}{l}\text { Referências } \\
\text { (números dos } \\
\text { documentos } \\
\text { selecionados) }\end{array}$ & $\begin{array}{l}\text { Indicadores utilizados nos estudos * } \\
\text { Indicador }\end{array}$ & $\begin{array}{c}n * * \\
(N=48)\end{array}$ & $\%$ \\
\hline \multirow[t]{36}{*}{$\begin{array}{l}\text { Desempenho dos } \\
\text { serviços de saúde }\end{array}$} & Efetividade & $\begin{array}{l}29,30,31,32,33,34,35 \\
47,48,49,50,51,52,53\end{array}$ & Taxa de mortalidade & 29 & 60,4 \\
\hline & & $54,55,56,57,58,59,60$ & Taxa de mortalidade por causa específica & 18 & 37,5 \\
\hline & & $\begin{array}{l}61,63,67,68,69,71,72, \\
75,77,78,79,80,82,83,\end{array}$ & Taxa de mortalidade global & 11 & 22,9 \\
\hline & & $86,93,95,96,97,98$ & Razão de óbitos observados/esperados & 6 & 12,5 \\
\hline & & & Proporção de óbitos evitáveis & 3 & 6,3 \\
\hline & & & Taxa de readmissão & 1 & 2,1 \\
\hline & & & Análise qualitativa & 2 & 4,2 \\
\hline & Adequação & $31,34,52,53,67,68,71$ & Taxa de adequação & 15 & 31,3 \\
\hline & & $\begin{array}{c}72,78,80,81,94,95,96 \\
97,98,101\end{array}$ & $\begin{array}{l}\text { Taxa de adequação do cuidado ao paciente com acidente } \\
\qquad \text { vascular cerebral } \star \star \star\end{array}$ & & \\
\hline & & & Taxa de adequação do cuidado ao paciente cardíaco *** & & \\
\hline & & & Taxa de adequação do cuidado à paciente em trabalho & & \\
\hline & & & de parto & & \\
\hline & & & Taxa de parto cesáreo & & \\
\hline & & & $\begin{array}{l}\text { Taxa de aplicação de protocolo recomendado em } \\
\text { situações específicas }\end{array}$ & & \\
\hline & & & Taxa de adequação do cuidado de enfermagem durante & & \\
\hline & & & a internação & & \\
\hline & & & Análise qualitativa & 1 & 2,1 \\
\hline & Eficiência & $30,35,51,64,65,67,73$ & Tempo de permanência & 7 & 14,6 \\
\hline & & $75,91,92,95,96,99$ & Taxa ou grau para eficiência & 3 & 6,3 \\
\hline & & & Taxa sintética de eficiência (estudos DEA) & & \\
\hline & & & Taxa de ocupação & & \\
\hline & & & Taxa de leitos disponíveis & & \\
\hline & & & Taxa de rotatividade & & \\
\hline & & & Relação entre saídas realizadas/contratadas & & \\
\hline & & & Relação de altas/internações & & \\
\hline & & & Relação funcionários/leito & & \\
\hline & & & Relação pacientes atendidos/funcionários & & \\
\hline & & & Custos por internação & 3 & 6,3 \\
\hline & Segurança & $32,54,62,63,64,65,66$ & Taxa de eventos adversos & 11 & 22,9 \\
\hline & & $\begin{array}{c}70,74,81,84,87,88 \\
89,98\end{array}$ & Erros de medicação \# & 4 & 8,3 \\
\hline & Respeito ao & $52,59,72,76,80,85,89$ & Taxa, grau ou nota para satisfação do paciente * & 5 & 10,4 \\
\hline & direito das & $90,94,96,100$ & Taxa sintética de satisfação & & \\
\hline & pessoas & & Informações sobre tratamentos alternativos & & \\
\hline & & & Informações sobre tempo de espera & & \\
\hline & & & $\begin{array}{l}\text { Reclamações sobre profissionais, atendimento ou estrutura } \\
\text { disponível }\end{array}$ & & \\
\hline & & & Análise qualitativa & 3 & 6,3 \\
\hline
\end{tabular}

(continua) 


\begin{tabular}{|c|c|c|c|c|c|}
\hline $\begin{array}{l}\text { Macrodimensão } \\
\text { (Viacava et al. 27) }\end{array}$ & $\begin{array}{c}\text { Dimensão } \\
\text { (Viacava et } \\
\text { al. 27) }\end{array}$ & $\begin{array}{l}\text { Referências } \\
\text { (números dos } \\
\text { documentos } \\
\text { selecionados) }\end{array}$ & $\begin{array}{l}\text { Indicadores utilizados nos estudos * } \\
\text { Indicador }\end{array}$ & $\begin{array}{c}n * * \\
(N=48)\end{array}$ & $\%$ \\
\hline Desempenho dos & Aceitabilidade & 58,59 & Análise qualitativa & 1 & 2,1 \\
\hline \multirow[t]{7}{*}{ serviços de saúde } & & & Informações sobre a adesão do paciente ao tratamento & & \\
\hline & & & recomendado & & \\
\hline & Acesso & $35,58,59,67,68,72,76$ & $\begin{array}{l}\text { Tempo entre indicação e realização de procedimento de } \\
\text { transferência entre setores ou estabelecimentos }\end{array}$ & 2 & 4,2 \\
\hline & & & Análise qualitativa & 3 & 6,3 \\
\hline & Continuidade & $54,58,59,67,76$ & Análise qualitativa & 4 & 8,3 \\
\hline & & & Barreiras administrativas cruzadas pelo paciente & & \\
\hline & & & $\begin{array}{l}\text { Informações sobre atendimento continuado com o mesmo } \\
\text { profissional }\end{array}$ & & \\
\hline \multirow[t]{7}{*}{ Estrutura \#\# } & Recursos & $\begin{array}{c}34,35,58,59,67,73,76 \\
95,96,97,98\end{array}$ & Taxa de adequação da estrutura & 6 & 12,5 \\
\hline & & & Medidas de adequação da estrutura física & & \\
\hline & & & Medidas de disponibilidade de equipamentos & & \\
\hline & & & Medidas de adequação de recursos humanos & & \\
\hline & & & Taxa de cancelamento de cirurgias por problemas de & & \\
\hline & & & estrutura & & \\
\hline & & & Taxa de rotatividade de funcionários & & \\
\hline
\end{tabular}

\footnotetext{
* Além dos indicadores calculados nos estudos aqui apresentados, as dimensões também foram exploradas a partir de análises qualitativas que não envolviam cálculo de indicadores ou a partir de estudos que incluíram aspectos relacionados a outras dimensões além do respeito ao direito das pessoas em taxas sintéticas de satisfação;

** Os estudos puderam ser classificados em mais de uma resposta conforme o conjunto de indicadores utilizados;

*** Inclui a indicação de procedimentos, exames ou prescrição de medicações recomendados e/ou tempo para sua realização;

\# Inclui erro de prescrição ou de administração de drogas ou procedimentos;

\#\# Apesar de não incluídos na estratégia de busca, alguns estudos de desempenho dos serviços também utilizaram indicadores relacionados à dimensão

"recursos", relacionada à estrutura do sistema de saúde.
}

como medida para ranqueamento de estabelecimentos segundo seu grau de desempenho e, em um deles 34, aplicada para a comparação entre períodos dos resultados obtidos em apenas um hospital. Importa destacar que, desses quatro estudos, a questão da necessidade de ajuste de risco da taxa de mortalidade foi discutida em 2 deles 29,30,31, e, nos outros 2 estudos, há mais de um documento publicado $32,33,34,35$, sendo que, em um deles 33,35 , a taxa de mortalidade é apresentada de maneira estratificada.

O ranqueamento de hospitais segundo níveis de qualidade foi apresentado em 16 estudos, enquanto 1 estudo publicou ranqueamento entre setores de um mesmo estabelecimento de saúde. Destaca-se que, entre os estudos que realizaram ranqueamento de estabelecimentos ou serviços, 3 aplicaram, para isso, taxas brutas de mortalidade sem ajuste de risco (Tabela 3 ).

Como fatores relacionados ao paciente, os estudos exploraram as variáveis: sexo, idade, raça/ cor, diagnóstico principal e secundário, índice de comorbidades, utilização de UTI, especialidade da internação (cirúrgica ou clínica), tipo de internação (eletiva ou urgência), uso de procedimentos selecionados, peso ao nascer, escala de risco, nível de severidade. Já em relação ao hospital, foram exploradas as variáveis: porte, volume médio de internações geral ou por causas específicas, natureza jurídica, esfera administrativa (municipal, estadual, federal ou privada), complexidade da assistência (baixa, média, alta), atividade de ensino, localização geográfica, perfil diagnóstico e demográfico dos pacientes, valores médios recebidos por internação, suficiência no financiamento, atendimento de emergência (sim ou não), características da estrutura física. Dessas variáveis, destacaram-se, por seu uso para estratificação de taxas, a idade, o sexo, o diagnóstico principal e uma medida de severidade ou escala de risco específica para o tema de estudo. 


\section{Discussão}

Esta revisão indica que a publicação de estudos sobre a qualidade do cuidado hospitalar no Brasil ganhou volume principalmente a partir do ano de 2004, tendo destaque aqueles que exploraram a abordagem do processo e do resultado do cuidado. Também se observou, no Brasil, a preocupação com a abordagem dos recursos da estrutura, justificada pela deficiência na oferta de profissionais e ambientes adequados à prestação dos cuidados, o que raramente ocorre em estudos de países desenvolvidos.

Em comparação de projetos de avaliação de qualidade adotados por diversos países, Groene et al. ${ }^{2}$ reportaram, como medidas da estrutura, os indicadores de governança responsiva, que medem a capacidade de resposta dos hospitais em relação às necessidades da população atendida, incluindo o redirecionamento de financiamento e recursos conforme os resultados obtidos. No Brasil, como dimensão da matriz conceitual do PRO-ADESS mais próxima ao conceito aplicado internacionalmente, está a "condução da estrutura do sistema de saúde" 26,27.

Apesar da falta de consenso mundial na delimitação das dimensões da qualidade e respectivas medidas, os temas mais frequentemente utilizados no mundo para a construção de indicadores de qualidade incluem a efetividade clínica, a segurança do paciente e a eficiência 1,2 que, aplicados à categorização proposta pelo PRO-ADESS, são medidas por meio de indicadores relacionados às dimensões adequação, segurança, efetividade e eficiência 26,27 . Conforme os resultados obtidos nesta revisão, tais dimensões mostraram-se as mais exploradas também em estudos brasileiros, seguindo a tendência observada nos estudos e projetos internacionais na área de avaliação da qualidade hospitalar.

A opção neste trabalho pela adoção da matriz conceitual de dimensões de avaliação desenvolvida pelo PRO-ADESS justifica-se, pois, apesar de basear-se em experiências internacionais como a canadense, a australiana e a inglesa, tal matriz apresenta, de maneira organizada, os principais elementos considerados essenciais para a melhor formulação de políticas no contexto da realidade brasileira 27 . No entanto, tendo em vista as correlações entre categorizações mundialmente aplicadas e a proposta do PRO-ADESS, as medidas aqui classificadas nas dimensões "efetividade" e "adequação" poderiam ser entendidas como medidas de efetividade clínica 36,37.

A predominância de estudos de corte transversal com o cálculo de indicadores baseado no uso de dados secundários, como se observou nesta revisão, não é inesperada, tendo em vista que a disponibilidade desse tipo de dados tem viabilizado a construção de indicadores de qualidade em todo o mundo ${ }^{2}$. No entanto, segundo a Agency for Healthcare Research and Quality (AHRQ) dos Estados Unidos 38, na construção de indicadores de qualidade, não basta que exista disponibilidade de dados, mas é imprescindível que haja evidência científica que suporte sua validade, precisão de medida (confiabilidade) e um mínimo de viés. Entre os desafios para o uso adequado de bases de dados secundárias, estão a garantia de sua cobertura (considerada o grau no qual o universo está registrado), sua completitude (grau de preenchimento dos campos), bem como sua consistência (grau em que variáveis relacionadas possuem valores coerentes) 39 .

Mas, para além dos desafios relacionados às fontes de dados utilizadas e ao crescente uso de indicadores de desempenho, são simultâneas preocupações de ordem metodológica, que trazem importantes consequências sobre as conclusões oriundas de estudos de avaliação da qualidade do cuidado. Destacam-se, entre essas preocupações: o caráter multidimensional e subjetivo do conceito de qualidade 40 ; a complexidade do cuidado médico na atualidade, envolvendo inúmeros processos especializados e com intensa incorporação tecnológica ${ }^{41}$; a confiabilidade dos indicadores de desempenho, ou seja, a correção nas aferições; a sua validade, ou o grau em que medem aquilo a que se pretendem medir com base no conhecimento científico a respeito, nesse caso, o impacto do cuidado no estado de saúde do paciente 8,39 ; bem como o perfil de casos (case mix) atendidos, relacionado ao consumo de recursos e ao prognóstico do paciente, um importante fator de confundimento na análise dos indicadores 42 .

Diversos estudos analisados nesta revisão aplicaram, a exemplo de estudos internacionais 43,44 , variáveis relacionadas ao paciente com a finalidade de ajustar risco; no entanto, muitos deles reportaram dificuldades com sua completitude e consistência. No que se refere ao hospital, assim como observado em estudos internacionais, o porte, o perfil diagnóstico dos pacientes e o volume de procedimentos vêm sendo frequentemente relacionados aos resultados de qualidade obtidos pelos hospitais e utilizados como variáveis de estratificação 45 .

A aplicação de medidas de mortalidade, adequação, eficiência e segurança na produção brasileira com vistas ao ranqueamento de hospitais aponta a necessidade de preocupação com a validade das medidas e com a utilização de métodos de ajuste para fatores de risco que podem interferir sobre o resultado do cuidado. Essas 
preocupações são especialmente importantes quando são comparados serviços, seja a título de transparência na informação, prestação de contas ou vinculação a mecanismos de pagamento, como vem se observando em todo o mundo, não apenas no setor hospitalar, mas também em ambientes ambulatoriais 8 .

Apesar da predominância de estudos metodologicamente cuidadosos com esse cenário, a produção brasileira ainda apresenta algumas falhas metodológicas na construção e apresentação de indicadores, principalmente na taxa de mortalidade, uma vez que análises com alto nível de agregação não padronizadas indicam problemas de validade 6,38. Em um ambiente competitivo que se baseie em medidas de desempenho publicadas com identificação dos hospitais, como vem sendo cada vez mais adotado no mundo 13,46 , tais falhas podem gerar impactos negativos injustos aos serviços.

É importante destacar que a aplicação dos resultados como insumo para mecanismos de pagamento de serviços hospitalares no Brasil não foi citada em quaisquer dos estudos analisados nesta revisão, ilustrando, por um lado, uma distância entre produção científica e tomada de decisão, mas, por outro lado, a blindagem da gestão financeira dos estabelecimentos em relação às fragilidades metodológicas observadas. No entanto, a natureza dos estudos selecionados para esta revisão exige a relativização dessa afirmação e não permite esgotar o tema do financiamento relacionado ao desempenho, uma vez que foram excluídos estudos direcionados à discussão da gestão hospitalar e da condução do sistema de saúde.

Nesse sentido, nas avaliações que tenham, como propósito, o monitoramento e a melhoria contínua da qualidade do cuidado ou a vinculação a mecanismos de pagamento por desempenho, é imprescindível definir, a priori, os aspectos a serem mensurados e os mecanismos metodológicos que garantam a validade dos indicadores calculados. Somente dessa forma pode-se esperar que os resultados desses estudos exerçam influência sobre o aperfeiçoamento do processo de cuidado, com avanços de efetividade e eficiência.

Tendo em vista os objetivos desta revisão, a qualidade dos estudos não foi aspecto decisivo para sua inclusão, portanto não foi aplicado nenhum critério ou instrumento para avaliação da qualidade metodológica. Pelo contrário, a inclusão de estudos independentemente de sua qualidade metodológica permitiu análises sobre as falhas atualmente cometidas em estudos que se propõem avaliar desempenho de hospitais, contribuindo para a visualização do cenário na- cional. Além disso, não se espera que os estudos incluídos apresentem viés de publicação, uma vez que foram encontrados resultados positivos e negativos, não se observando uma preferência pela publicação de um ou de outro.

Os principais limites da presente revisão estão relacionados à exclusão de documentos governamentais, relatórios de pesquisa, livros, textos para discussão, publicações não indexadas nas bases de dados bibliográficas consultadas ou indexadas por outro descritor que não os incluídos na equação de busca, além das teses e dissertações publicadas internacionalmente, portanto não alcançáveis pelas estratégias de busca adotadas.

A restrição de acesso à base Embase também pode limitar os achados deste estudo. Contudo, entende-se que os principais artigos científicos voltados para a realidade brasileira na área da avaliação em saúde encontram-se indexados nas bases bibliográficas consultadas. Além disso, esforços foram empreendidos para minimizar omissões, tal como a análise de dissertações de mestrado e teses de doutorado e a consulta às referências bibliográficas das publicações.

Outra limitação relaciona-se à possibilidade de erro nas classificações dos estudos realizadas pelos autores, uma vez que utilizaram, para essa tarefa, exclusivamente os textos disponíveis nos documentos publicados.

\section{Conclusão}

Esta revisão identificou um aumento recente na produção acadêmica nacional sobre a qualidade do cuidado hospitalar. Contudo, comparativamente ao volume de artigos disponíveis no cenário internacional, no Brasil, a produção é ainda restrita em volume e escopo das análises realizadas.

Ainda assim e mesmo considerando que apenas recentemente órgãos governamentais ou agências reguladoras têm assumido compromissos nessa área e divulgado o resultado de avaliações de desempenho, o panorama apresentado neste trabalho traz importantes elementos para o debate sobre a qualidade do serviço hospitalar no país. Evidenciam-se, sobretudo, quais lacunas, problemas e desafios são necessários superar para dar continuidade a esse tipo de avaliação no país, contribuindo com seus resultados para o redirecionamento de recursos e a definição de novas estratégias de gestão e de políticas inovadoras baseadas no conhecimento produzido.

Tal cenário não reduz a importância dos esforços realizados até aqui, mas aponta para a necessidade do desenvolvimento de estratégias 
que viabilizem o uso das informações obtidas por meio desse tipo de abordagem para aumentar o efeito positivo sobre a qualidade do cuidado e desse sobre o estado de saúde dos pacientes, com reflexos importantes sobre o desempenho e a efetividade dos serviços.

\section{Resumen}

La evaluación de la calidad de los servicios hospitala rios está recibiendo cada vez más atención internacional, dirigida por la demanda de inversores (fundadores), proveedores, profesionales y pacientes. El objetivo de este estudio es revisar la literatura de los estudios de evaluación, realizados sobre la calidad de la atención hospitalaria en Brasil, y analizar los enfoques importantes, la metodología y los indicadores aplicados. El diseño de la investigación fue una revisión sistemática de artículos científicos, de tesis de Ph.D. y de M.Sc. con análisis empírico, publicados entre 1990 y 2011. Se identificaron 2.169 documentos de los cuales se incluyeron 62 en la revisión, que representan 48 estudios separados. Principalmente, se utilizaron fuentes secundarias de datos con el análisis de las dimensiones de efectividad, adecuación, seguridad y eficiencia, enfatizando en la aplicación de las tasas de mortalidad, de adecuación y de eventos adversos, y de la duración de la estadía. Fueron aplicados métodos para controlar la diferencia en el riesgo personal de pacientes. Esta revisión señala elementos centrales con el fin de contribuir para el desarrollo del tema en el país y la mejora de la calidad de la asistencia sanitaria prestada.

Evaluación de la Calidad de Atención de Salud; Servicios Hospitalarios; Indicadores de Calidad de la Atención de Salud

\section{Colaboradores}

Todos os autores participaram da concepção do estudo, aquisição, análise e interpretação dos dados, bem como da aprovação da versão a ser publicada. J. P. Machado realizou a redação do artigo e sua revisão final. A. C. M. Martins contribuiu para a redação do artigo e realizou sua revisão crítica. M. S. Martins contribuiu para a redação do artigo e realizou sua revisão crítica.

\section{Agradecimentos}

Agradecemos às professoras Rosângela Caetano e Claudia Travassos pela importante contribuição principalmente na definição das estratégias de busca e na apresentação dos resultados. 


\section{Referências}

1. Fung V, Schmittidiel JA, Fireman B, Meer A, Thomas $\mathrm{S}$, Smider N, et al. Meaningful variation in performance: a systematic review. Med Care 2010; 48:140-8.

2. Groene O, Skau JKH, Frølich A. An international review of projects on hospital performance assessment. Int J Qual Health Care 2008; 20:162-71.

3. Mulley AG. Inconvenient truths about supplier induced demand and unwarranted variation in medical practice. BMJ 2009; 339:b4073.

4. Vecina-Neto G, Malik AM. Tendências na assistência hospitalar. Ciênc Saúde Coletiva 2007; 12: 825-39.

5. Basinski ASH, Naylor CD, Ferris LE, Willians JI, Llewellyn-Thomas HA, Cohen MM. Quality of care: 1. What is quality and how can it be measured? Health Services Research Group. Can Med Assoc J 1992; 146:2153-8.

6. Travassos C, Noronha J, Martins M. Mortalidade hospitalar como indicador de qualidade: uma revisão. Ciênc Saúde Coletiva 1999; 4:367-81.

7. Chassin MR, Park RE, Lohr KN, Keesey J, Brook RH. Differences among hospitals in Medicare patient mortality. Health Serv Res 1989; 24:1-31.

8. Chassin MR, Loeb JM, Schmaltz SP. Accountability measures - using measurement to promote quality improvement. New Engl J Med 2010; 363:683-8.

9. Gouvêa CSD, Travassos C. Indicadores de segurança do paciente para hospitais de pacientes agudos: revisão sistemática. Cad Saúde Pública 2010; 26:1061-78.

10. Klassen A, Miller A, Anderson N, Shen J, Schiariti V, O'Donnell M. Performance measurement and improvement frameworks in health, education and social services systems: a systematic review. Int J Qual Health Care 2009; 22:44-69.

11. Kelley E, Hurst J. Health care quality indicators project: conceptual framework paper. Paris: Organisation for Economic Co-operation and Development; 2006. (OECD Health Working Papers, 23).

12. Jacobson B, Mindell J, McKee M. Hospital mortality league tables. BMJ 2003; 326:777-8.

13. Nunes R, Brandão C, Rego G. Public accountability and sunshine healthcare regulation. Health Care Anal 2011; 19:352-64.

14. Kondilis E, Gavana M, Giannakopoulos S, Smyrnakis E, Dombros N, Benos A. Payments and quality of care in private for-profit and public hospitals in Greece. BMC Health Serv Res 2011; 11:234.

15. Feldman LB, Gatto MAF, Cunha ICKO. História da evolução da qualidade hospitalar: dos padrões à acreditação. Acta Paul Enferm 2005; 18:213-9.

16. Noronha JC, Rosa MLG. Country report. Quality of healthcare: growing awareness in Brazil. Int J Qual Health Care 199; 11:437-41.

17. Noronha JC, Travassos CM, Rosa ML. Quality improvement initiatives in Brazil: a progress report. Jt Comm J Qual Improv 1999; 25:565-73.

18. Noronha JC, Pereira TR. Health care reform and quality initiatives in Brazil. Jt Comm J Qual Improv 1998; 24:251-63.
19. Ministério da Saúde. Portaria GM/MS no 382, de 10 de março de 2005. Institui o Programa Nacional de Avaliação de Serviços de Saúde - PNASS e dá outras providências. 2005. Diário Oficial da União 2005; 10 mar.

20. Coordenação-Geral de Monitoramento e Avaliação, Departamento de Monitoramento e Avaliação do SUS, Secretaria Executiva, Ministério da Saúde. Programa de avaliação para a qualificação do Sistema Único de Saúde. http://portal.saude. gov.br/portal/arquivos/pdf/programa_avaliacao_ qualificacao_SUS_versao3.pdf (acessado em 20/ Jan/2012).

21. Comitê Técnico Assessor para Acompanhamento do Programa de Avaliação para a Qualificação do SUS, Ministério da Saúde. Î́ndice de desempenho do Sistema Único de Saúde. Brasília: Ministério da Saúde; 2011.

22. Agência Nacional de Saúde Suplementar. Programa de qualificação da saúde suplementar: qualificação das operadoras. http://www.ans.gov.br/ima ges/stories/A_ANS/Transparencia_Institucional/ Indicadores_de_qualidade/texto_base_aval_des_ idss_20090811.pdf (acessado em 20/Jan/2012).

23. Agência Nacional de Vigilância Sanitária. Segurança do paciente e qualidade em serviços de saúde. Boletim Informativo 2011; 1:1-12.

24. Agência Nacional de Vigilância Sanitária. Segundo relatório sobre o sistema brasileiro de acreditação. Gerência-Geral de Tecnologia de Serviços de Saúde. http://www.anvisa.gov.br/servicosaude/ avalia/projetos/rel_acredita2.pdf (acessado em 20/ Jan/2012).

25. Malik AM. Quality improvement issues in Brazil. Jt Comm J Qual Improv 1997; 23:55-9.

26. Viacava F, Almeida C, Caetano R, Fausto M, Macinko J, Martins M, et al. Uma metodologia de avaliação do desempenho do sistema de saúde brasileiro. Ciênc Saúde Coletiva 2004; 9:711-24.

27. Viacava F, Ugá MAD, Porto S, Laguardia J, Moreira RS. Avaliação de desempenho de sistemas de saúde: um modelo de análise. Ciênc Saúde Coletiva 2012; 17:921-34.

28. Donabedian A. Basic approaches to assessment: structure, process and outcome. In: Donabedian A, editor. The definition of quality and approaches to its assessment. Ann Arbor: Health Administration Press; 1980. p. 77-128.

29. Piegas LS, Haddad N. Intervenção coronariana percutânea no Brasil: resultados do Sistema Único de Saúde. Arq Bras Cardiol 2011; 96:317-24.

30. Piegas LS, Bittar OJ, Haddad N. Myocardial revascularization surgery (MRS): results from national health system (SUS). Arq Bras Cardiol 2009; 93:555-60.

31. Gouvêa CS, Travassos C, Fernandes C. Produção de serviços e qualidade da assistência hospitalar no Estado do Rio de Janeiro, Brasil - 1992 a 1995. Rev Saúde Pública 1997; 31:600-17.

32. Oliveira MI, Dias MA, Cunha CB, Leal MC. Qualidade da assistência ao trabalho de parto pelo Sistema Único de Saúde, Rio de Janeiro (RJ), 19992001. Rev Saúde Pública 2008; 42:895-902. 
33. Godoy PH, Klein CH, Souza-e-Silva NA, Oliveira GM. Letalidade hospitalar nas angioplastias coronárias no Estado do Rio de Janeiro, Brasil, 19992003. Cad Saúde Pública 2007; 23:845-51.

34. Rocha AS, Araújo MP, Volscham A, Carvalho LA, Ribeiro A, Mesquita ET. Evidence of healthcare quality improvement in acute myocardial infarction. Arq Bras Cardiol 2010; 94:681-4.

35. Mesquita ET, Ribeiro A, Araújo MP, Campos LAA, Fernandes MA, Colafranceschi AS, et al. Indicadores de qualidade assistencial na cirurgia de revascularização miocárdica isolada em centro cardiológico terciário. Arq Bras Cardiol 2008; 90:350-4.

36. Veillard J, Champagne F, Klazinga N, Kazandjian V, Arah OA, Guisset AL. A performance assessment framework for hospitals: the WHO Regional Office for Europe PATH project. Int J Qual Health Care 2005; 17:487-96.

37. Institute of Medicine. Crossing the quality chasm: a new health system for the $21^{\text {st }}$ century. Committee on Quality Health Care in America, 2001. http://www.nap.edu/catalog/10027.html (acessado em 20/Jan/2012).

38. Agency for Healthcare Research and Quality. Quality indicators - guide to inpatient quality indicators: quality of care in hospitals - volume, mortality, and utilization. Rockville: Agency for Healthcare Research and Quality; 2002.

39. Lima CRA, Schramm JMA, Coeli CM, Silva MEM. Revisão das dimensões de qualidade dos dados e métodos aplicados na avaliação dos sistemas de informação em saúde. Cad Saúde Pública 2009; 25:2095-109.

40. Donabedian A. The definition of quality: a conceptual exploration. In: Donabedian A, editor. The definition of quality and approaches to its assessment. Ann Arbor, Michigan: Health Administration Press; 1980. p. 1-31.

41. Frias PG, Costa JMBS, Figueiró AC, Mendes MFM, Vidal SA. Atributos da qualidade em saúde. In: Samico I, Felisberto E, Figueiró AC, Frias PG, organizadores. Avaliação em saúde. Bases conceituais e operacionais. Rio de Janeiro: MedBook; 2010. p. 43-55.

42. Martins M, Portela MC, Noronha MF. Desempenho hospitalar: avaliação do tempo de permanên cia e mortalidade entre prestadores no Estado do Rio de Janeiro. In: Ugá MAD, Sá MC, Martins M, Braga Neto FC, organizadores. A gestão do SUS no âmbito estadual: o caso do Rio de Janeiro. Rio de Janeiro: Editora Fiocruz; 2010. p. 241-78.

43. Van Den Bosch WF, Spreeuwenberg P, Wagne C. Variations in hospital standardised mortality ratios (HSMR) as a result of frequent readmissions. BMC Health Serv Res 2012; 12:91.

44. Pitches DW, Mohammed MA, Lilford RJ. What is empirical evidence that hospitals with higher-risk adjusted mortality rates provide poorer quality care? A systematic review of the literature. BMC Health Serv Res 2007; 7:91-8.

45. Lehrman WG, Elliot MN, Goldstein E, Beckett M, Klein DJ, Giordano LA. Characteristics of hospitals demonstrating superior performance in patient experience and clinical process measures of care. Med Care Res Rev 2010; 67:38-55.
46. Fisher ES, Shortell SM. Accountable care organizations: accountable for what, to whom and how. JAMA 2010; 304:1715-6.

47. Gomes AS. Mortalidade hospitalar: modelos preditivos de risco usando os dados do sistema de informações hospitalares do SUS [Tese de Doutorado]. Porto Alegre: Programa de Pós-graduação em Epidemiologia, Universidade Federal do Rio Grande do Sul; 2009.

48. Gomes AS, Klück MM, Riboldi J, Fachel JMG. Modelo preditivo de óbito a partir de dados do Sistema de Informações Hospitalares. Rev Saúde Pública 2010; 44:934-41.

49. Gomes AS, Klück MM, Fachel JMG, Riboldi J. Fatores associados à mortalidade hospitalar na rede SUS do Rio Grande do Sul, em 2005: aplicação de modelo multinível. Rev Bras Epidemiol 2010; 13:533-42.

50. Iucif Jr. N, Rocha JSY. Estudo da desigualdade na mortalidade hospitalar pelo índice de comorbidade de Charlson. Rev Saúde Pública 2004; 38:780-6.

51. Martins M, Blais R, Leite IC. Mortalidade hospitalar e tempo de permanência: comparação entre hospitais públicos e privados na região de Ribeirão Preto, São Paulo, Brasil. Cad Saúde Pública 2004; 20 Suppl 2:S268-82.

52. Rolim CLRC. Avaliação da efetividade do tratamento hospitalar do acidente vascular cerebral agudo no Sistema Único de Saúde - SUS - utilização da mortalidade hospitalar como indicador de desempenho [Dissertação de Mestrado]. Rio de Janeiro: Escola Nacional de Saúde Pública Sergio Arouca, Fundação Oswaldo Cruz; 2009.

53. Rolim CLRC, Martins M. Qualidade do cuidado ao acidente vascular cerebral isquêmico no SUS. Cad Saúde Pública 2001; 27:2106-16.

54. Daud-Gallotti R, Novaes HMD, Lorenzi MC, ElufNeto J, Okamura MN, Velasco IT. Adverse events and death in stroke patients admitted to the emergency department of a tertiary university hospital. Eur J Emerg Med 2005; 12:63-71.

55. Noronha JC. Utilização de indicadores de resultados para a avaliação da qualidade em hospitais de agudos: mortalidade hospitalar após cirurgia de revascularização do miocárdio em hospitais brasileiros [Tese de Doutorado]. Rio de Janeiro: Institu to de Medicina Social, Universidade do Estado do Rio de Janeiro; 2001.

56. Noronha JC, Travassos C, Martins M, Campos MR, Maia P, Panezzuti R. Avaliação da relação entre volume de procedimentos e a qualidade do cuidado: o caso de cirurgia coronariana no Brasil. Cad Saúde Pública 2003; 19:1781-9.

57. Noronha JC, Martins M, Travassos C, Campos MR, Maia P, Panezzuti R. Aplicação da mortalidade hospitalar após a realização de cirurgia de revascularização do miocárdio para monitoramento do cuidado hospitalar. Cad Saúde Pública 2004; 20 Suppl 2:S322-30.

58. Paiva SMA. Qualidade da assistência hospitalar: avaliação da satisfação dos usuários durante seu período de internação [Tese de Doutorado]. Ribeirão Preto: Escola de Enfermagem de Ribeirão Preto, Universidade de São Paulo; 2006. 
59. Paiva SM, Gomes EL. Assistência hospitalar: avaliação da satisfação dos usuários durante seu período de internação. Rev Latinoam Enferm 2007; 15:973-9.

60. Oliveira TML, Oliveira GMM, Klein CH, Silva NAS, Godoy PH. Letalidade e complicações da cirurgia de revascularização miocárdica no Rio de Janeiro, de 1999 a 2003. Arq Bras Cardiol 2010; 95:303-12.

61. Kelles SM, Barreto SM, Guerra HL. Mortality and hospital stay after bariatric surgery in 2,167 patients: influence of the surgeon expertise. Obes Surg 2009; 19:1228-35.

62. Mendes W, Martins M, Rozenfeld S, Travassos C. The assessment of adverse events in hospitals in Brazil. Int J Qual Health Care 2009; 21:279-84.

63. Martins M, Travassos C, Mendes W, Pavão AL. Hospital deaths and adverse events in Brazil. BMC Health Serv Res 2011; 11:223.

64. Moura CS, Acurcio FA, Belo NO. Drug-drug interactions associated with length of stay and cost of hospitalization. J Pharm Pharm Sci 2009; 12:266-72.

65. Moura C, Prado N, Acurcio F. Potential drug-drug interactions associated with prolonged stays in the intensive care unit: a retrospective cohort study. Clin Drug Investig 2001; 31:309-16.

66. Nascimento CCP, Toffoletto MC, Gonçalves LA, Freitas WG, Padilha KG. Indicadores de resultados da assistência: análise dos eventos adversos durante a internação hospitalar. Rev Latinoam Enferm 2008; 16:746-51.

67. Rosa ML, Hortale VA. Avoidable perinatal deaths and obstetric health care structure in the public health care system: a case study in a city in greater metropolitan Rio de Janeiro. Cad Saúde Pública 2000; 16:773-83.

68. Chiavone PA, Rasslan S. Influence of time elapsed from end of emergency surgery until admission to intensive care unit, on Acute Physiology and Chronic Health Evaluation II (APACHE II) prediction and patient mortality rate. São Paulo Med J 2005; 123:167-74.

69. Amaral ACS, Coeli CM, Costa MCE, Cardoso VS, Toledo ALA, Fernandes CR. Perfil de morbidade e de mortalidade de pacientes idosos hospitalizados. Cad Saúde Pública 2004; 20:1617-26.

70. Dias MAE. Segurança do paciente: rastreamento de resultados adversos no Sistema de Informação Hospitalar (SIH-SUS) [Dissertação de Mestrado]. Rio de Janeiro: Escola Nacional de Saúde Pública Sergio Arouca, Fundação Oswaldo Cruz; 2010.

71. Escosteguy CC, Portela MC, Vasconcellos MT, Medronho RA. Pharmacological management of acute myocardial infarction in the municipal district of Rio de Janeiro. São Paulo Med J 2001; 119:193-9.

72. Borges JBC, Carvalho SMR, Silva MAM. Qualidade do serviço prestado aos pacientes de cirurgia cardíaca do Sistema Único de Saúde-SUS. Rev Bras Cir Cardiovasc 2010; 25:172-82.

73. Cesconetto A, Lapa JS, Calvo MCM. Avaliação da eficiência produtiva de hospitais do SUS de Santa Catarina, Brasil. Cad Saúde Pública 2008; 24 : 2407-17.

74. Costa LA, Loureiro S, Oliveira MGG. Errores de medicación de dos hospitales de Brasil. Farm Hosp 2006; 30:235-9.
75. Gonçalves AC, Noronha CP, Lins MPE, Almeida RMVR. Data envelopment analysis for evaluating public hospitals in Brazilian state capitals. Rev Saúde Pública 2007; 41:427-35.

76. Kotaka F, Pacheco MLR, Higaki Y. Avaliação pelos usuários dos hospitais participantes do programa de qualidade hospitalar no Estado de São Paulo, Brasil. Rev Saúde Pública 1997; 31:171-7.

77. Lansky S, França E, Leal MC. Mortes perinatais evitáveis em Belo Horizonte, Minas Gerais, Brasil, 1999. Cad Saúde Pública 2002; 18:1389-400.

78. Lansky S, França E, César CC, Neto LCM, Leal MC. Mortes perinatais e avaliação da assistência ao parto em maternidades do Sistema Único de Saúde em Belo Horizonte, Minas Gerais, Brasil, 1999. Cad Saúde Pública 2006; 22:117-30.

79. Lansky S, Subramanian SV, França E, Kawachi I. Higher perinatal mortality in National Public Health System hospitals in Belo Horizonte, Brazil, 1999: a compositional or contextual effect? BJOG 2007; 14:1240-5.

80. Oliveira MI, Dias MA, Cunha CB, Leal MC. Qualidade da assistência ao trabalho de parto pelo Sistema Único de Saúde, Rio de Janeiro (RJ), 19992001. Rev Saúde Pública 2008; 42:895-902.

81. Louro E, Romano-Lieber NS, Ribeiro E. Eventos adversos a antibióticos em pacientes internados em um hospital universitário. Rev Saúde Pública 2007; 41:1042-8.

82. Masella CA, Pinho VF, Costa Passos AD, Spencer Netto FA, Rizoli S, Scarpelini S. Temporal distribution of trauma deaths: quality of trauma care in a developing country. J Trauma 2008; 65:653-8.

83. Matos GC, Rozenfeld S, Martins M. Human albumin use at hospitals in the Metropolitan Region of Rio de Janeiro, Brazil. Cad Saúde Pública 2010; 26:981-90

84. Passarelli MC, Jacob-Filho W, Figueras A. Adverse drug reactions in an elderly hospitalised population: inappropriate prescription is a leading cause. Drugs Aging 2005; 22:767-77.

85. Ricci NA, Wanderley FS, Oliveira MS, Rebelatto JR. O hospital-escola de São Carlos: análise do funcionamento por meio da satisfação dos usuários. Ciênc Saúde Coletiva 2011; 16 Suppl 1:1125-34.

86. Schramm JMA, Szwarcwald CL, Esteves MAP. Assistência obstétrica e risco de internação na rede de hospitais do Estado do Rio de Janeiro. Rev Saúde Pública 2002; 36:590-7.

87. Silva AEBC, Reis AMM, Miasso AI, Santos JO, Cassiani SHB. Eventos adversos a medicamentos em um hospital sentinela do Estado de Goiás, Brasil. Rev Latinoam Enferm 2011; 19:378-86.

88. Carneiro FS, Bezerra ALQ, Silva AEBC, Souza LP, Paranaguá TTB, Branquinho NCSS. Eventos adversos na clínica cirúrgica de um hospital universitário: instrumento de avaliação da qualidade. Rev Enferm UERJ 2011; 19:204-11.

89. Souza LP, Bezerra ALQ, Silva AEBC, Carneiro FS, Paranaguá TTB, Lemos LF. Eventos adversos: instrumento de avaliação do desempenho em centro cirúrgico de um hospital universitário. Rev Enferm UERJ 2011; 19:127-33. 
90. Cruz WBS. Análise dos níveis de satisfação dos usuários de um hospital privado [Dissertação de Mestrado]. São Paulo: Faculdade de Enfermagem, Universidade de São Paulo; 2008.

91. Marinho A. Estudo de eficiência em hospitais públicos e privados com a geração de "rankings". Rev Adm Pública 1998; 32:145-58.

92. Zucchi P. Funcionários por leito: estudo em alguns hospitais públicos e privados. Rev Adm Pública 1998; 32:65-76.

93. Martins MS. Mortalidade hospitalar: novas abordagens metodológicas [Dissertação de Mestrado] Rio de Janeiro: Escola Nacional de Saúde Pública, Fundação Oswaldo Cruz; 1991.

94. Costa MTF. Avaliação da assistência em internações de hospital pediátrico no Município do Rio de Janeiro: discussão sobre uma abordagem metodológica [Dissertação de Mestrado]. Rio de Janeiro: Escola Nacional de Saúde Pública, Fundação Oswaldo Cruz; 1994.

95. Gonçalves FANI. Infarto agudo do miocárdio em idosos: morbimortalidade e indicadores de desempenho dos serviços hospitalares no Brasil [Dissertação de Mestrado]. Rio de Janeiro: Instituto de Medicina Social, Universidade do Estado do Rio de Janeiro; 2005.

96. Golovattei MAR. Associação da medida de satisfação dos usuários com indicadores de produção e de qualidade assistencial em hospitais estaduais de São Paulo [Dissertação de Mestrado]. São Paulo: Escola de Administração de Empresas de São Paulo, Fundação Getúlio Vargas; 2010.
97. Alves MTSSB. Avaliação da qualidade da assistência ao parto hospitalar no Sistema Único de Saúde em São Luís [Dissertação de Mestrado]. São Luís: Mestrado em Saúde e Ambiente, Universidade Federal do Maranhão; 1999.

98. Costa MP. Avaliação de qualidade em unidade de tratamento intensivo neonatal no Sul do Brasil [Dissertação de Mestrado]. Rio Grande: Mestrado em Ciências da Saúde, Universidade Federal do Rio Grande; 2010.

99. Haddad N. Duração média de internação e mortalidade hospitalar de pacientes coronarianos submetidos à cirurgia de revascularização do miocárdio em hospital especializado em cardiologia. Rev Adm Saúde 2008; 10:29-32.

100. Santos SR, Lacerda MC. Fatores de satisfação e insatisfação em pacientes atendidos pelo SUS. Rev Bras Enferm 1999; 52:43-53.

101. d'Orsi E, Chor D, Giffin K, Angulo-Tuesta A, Barbosa GP, Gama AS, et al. Qualidade da atenção ao parto em maternidades do Rio de Janeiro. Rev Saúde Pública 2005; 39:645-54.

102. Guerra HL, Giatti L, Lima-Costa MF. Mortalidade em internações de longa duração como indicador de qualidade da assistência hospitalar ao idoso. Epidemiol Serv Saúde 2004; 13:247-53.

Recebido em 14/Out/2012

Versão final reapresentada em 06/Fev/2013

Aprovado em 04/Mar/2013 